\title{
Mini-endovascular aneurysm repair: a minimalist approach for a minimally invasive procedure
}

\author{
Ahmet Unlu, Ahmet Baris Durukan \\ Department of Cardiovascular Surgery, Salihli Private MediGüneş Hospital, Salihli, Manisa, Turkey \\ Kardiochir Torakochir Pol 2021; 18 (1): 50-54
}

\begin{abstract}
Introduction: Abdominal aortic aneurysms represent the majority of all aneurysms of the aorta. Endovascular aneurysm repair (EVAR) is an alternative procedure to surgical repair. Although general and regional anaesthesia are frequently used during EVAR procedures, local anaesthesia has become one of the anaesthesia options for which there is increasing experience.

Aim: We reported our EVAR cases in which we routinely used femoral local anaesthesia.

Material and methods: Between August 2016 and June 2020, the EVAR procedure was applied to 22 infrarenal abdominal aortic aneurysm patients under femoral local anaesthesia. Open femoral artery access through a groin incision was used in all patients. Patients were followed up for graft- and wound-related complications.

Results: The mean age of the patients was $72.59 \pm 6.6$ years (min: 60, max: 84 ). Mean aneurysm sac diameter was 61.04 $\pm 8.76 \mathrm{~mm}$. Bifurcated stent graft was used in 21 (95.5\%) patients. An aorto-uni-iliac stent graft was used for 1 (4.5\%) patient due to contralateral total iliac occlusion. Endoleak was observed in 6 patients. In-hospital mortality was observed in 2 patients; both cases were ruptured with haemodynamic instability (9\%). Revision in the groin area was performed in 3 (13.6\%) patients due to local wound complications.

Conclusions: Although the EVAR procedure has been described as a safer and more easily applicable alternative to surgical repair, it is disadvantageous in terms of increasing treatment costs. Anaesthesia preference and incision size with a more minimalist approach can reduce the length of hospital stay and minimize the complications that may occur after the procedure, resulting in decreased costs.
\end{abstract}

Key words: abdominal aortic aneurysm, stents, vascular grafting, anaesthesia, local.

\section{Introduction}

Abdominal aortic aneurysms (AAA) represent the majority of all aneurysms of the aorta, and the mortality rate for a ruptured AAA is very high [1]. For decades, open surgical techniques were performed for treatment. Parodi et al. first described endovascular aneurysm repair (EVAR) as a less invasive and safer intervention for AAA repair in the early 1990s [2]. Over the years, EVAR became the first-line treatment option for infrarenal AAAs [3-5]. The selection of anaesthetic technique during an EVAR procedure depends on the experience of the operator, the complexity of the aneurysm, and the comorbidities of the patient, but there is still a lack of evidence of which technique is superior [1]. Frequently, regional anaesthesia (RA) or general anaesthesia (GA) are used for EVAR procedures. It can also be performed by femoral local anaesthesia (LA). Early mobilization, and very short intensive care unit (ICU) and hospital stay are the main advantages of LA.

\section{Aim}

In this study, we reported our experience of EVAR procedures for infrarenal AAAs performed by routine femoral LA.

\section{Material and methods}

Between August 2016 and June 2020, 24 patients with infrarenal AAA were admitted to our hospital. We are working as one of the 2 clinics serving a population of 220,000 in total. Therefore, the number of patients admitted to our clinic with a diagnosis of infrarenal AAA was limited; we included all patients to the study. Computed tomography angiography (CTA) was used for the diagnosis of AAA in all patients. Two patients underwent open repair, 1 was deemed unfit for EVAR procedure because of anatomical incompatibility and the other due to development of retrograde iatrogenic dissection during the EVAR procedure.

A standard protocol including blood tests and detailed cardiovascular assessment were done 1 day prior to hospi-

Address for correspondence: Prof. Ahmet Baris Durukan, Department of Cardiovascular Surgery, Salihli Private MediGüneş Hospital, Salihli, Manisa/Turkey, phone: +905322273814, e-mail: barisdurukan@yahoo.com

Received: 25.10.2020, accepted: 15.02.2021. 
talization. Anti-hypertensive therapy was initiated if needed before the EVAR procedure. The EVAR procedure was performed on the remaining 22 patients under $L A$. There were no contraindications for LA, like advanced obesity, infection in the groin area, severe atherosclerotic lesions in the common femoral artery, or any previous interventions that would prevent re-intervention. In all cases, the anaesthesiologist and suitable technical infrastructures were present in the angiography unit in case of a possible change in anaesthesia technique. There was no conversion from LA to GA or RA. Two 18G cannulas were used in all patients for peripheral IV access. Radial artery catheterization was performed for haemodynamic monitorization. Open femoral artery access through a groin incision was used in all patients. $10 \mathrm{ml}$ of prilocaine hydrochloride (Pricalest, INC Pharma ${ }^{\circledR}$, İstanbul/Turkey) was used for each groin for femoral LA. Following anaesthetic application, a bilateral 4-cm vertical incision was made and the femoral arteries were explored. 7F sheaths were placed in each of the femoral arteries by Seldinger technique. $5000 \mathrm{U}$ of heparin (Vasparin, VEM Medicine ${ }^{\circledR}$, Tekirdağ/Turkey) was given intravenously before deployment the stent graft, and activated clotting time (ACT) test was applied intermittently to monitor the heparin dosage, maintaining an ACT of over $200 \mathrm{~s}$. Endurant II Stent Graft System (Medtronic Inc., Minneapolis, USA) was the only stent graft used for all cases. Strict haemodynamic follow-up monitoring was carried out during EVAR procedure. All patients were followed up in the ICU for 2 to 3 hours until sustained haemodynamic stabilization was observed. Surgical mini-Hemovac drains were routinely placed after haemostasis was achieved in the groin area. The patients were discharged after acceptable drainage from Hemovac drains was observed. Patients were followed up for wound- and graft-related complications such as haematoma, lymphocele, wound infection, and endoleak and graft infection, from August 2016 to August 2020.

\section{Statistical analysis}

The data were analysed using SPSS version 17 software (Statistical Package for the Social Sciences Inc., Chicago, IL, USA). Continuous variables were presented as "mean \pm SD" and median (min., max.), and categorical variables were presented as numbers and percentages.

\section{Results}

All patients were male. The mean age of the patients was $72.59 \pm 6.6$ years (min.: 60, max.: 84). Hypertension was present in 21 (95.5\%) patients, hyperlipidaemia in 13 (59\%) patients, and diabetes mellitus in 4 (18.1\%) patients. Coronary artery disease (CAD) was detected in 13 (59\%) patients. The mean aneurysm sac diameter was $61.04 \pm 8.76$ mm (min.: 41, max.: 81). Infrarenal AAA was present in 20 (91\%) Patients. Two (9\%) patients had saccular aneurysm (1 of them had concomitant dissection). Two (9\%) patients had ruptured infrarenal AAA and were haemodynamically stable. The preoperative characteristics of the patients are summarized in Table I.
The type of stent graft varied according to anatomy and pathology. Bifurcated stent graft was used in 21 (95.5\%) patients. We also used an aorto-uni-iliac stent graft for $1(4.5 \%)$ patient due to contralateral total iliac occlusion (Figure 1). After adding sedative anaesthesia to LA, extra anatomic femorofemoral bypass surgery using a prosthetic vascular graft was performed after the EVAR procedure in order to provide blood supply to the contralateral leg. Due to the iliac extension of the AAA, we performed right iliac extension for 5 (22\%) patients and left iliac extension for 2 (9\%) patients. The mean operative time was 107.4 \pm 17.36 min (min.: 83, max.: 143). Table II shows the intraoperative features of the patients.

In postoperative period, mean hospital stay was 3.45 \pm 1.05 days (min.: 2, max.: 6). Endoleak was observed in 6 patients (3 patients type $1 \mathrm{a}: 13.6 \%$, 1 patient type $1 \mathrm{~b}: 4.5 \%$ and 2 patients type 2: $9 \%$ ), type 1 endoleaks were treated by balloon dilatation immediately after the procedure. Type 2 endoleaks were not intervened due to the absence of aneurysm sac expansion throughout the follow-up. Inhospital mortality was observed in 2 (9\%) patients who were emergently intervened due to ruptured AAA. They died in the early postoperative period due to accompanying cardiovascular comorbidities. One of them had a previous coronary by-pass surgery and reduced ventricular function with severe dilated cardiomyopathy and died of an anteroseptal myocardial infarction after the procedure. The other patient had chronic renal impairment and died due to acute renal failure. Revision in the groin area was performed in 3 (13.6\%) patients because of local wound complications such as lymphocele and haematoma. Mean follow-up time was $16.05 \pm 11.48$ (min.: 3, max.: 36) months, and no mortality or further complications regarding graft or wound were observed throughout the follow-up period. The postoperative characteristics of the patients are given in Table III.

Table I. Preoperative characteristics of patients

\begin{tabular}{lc} 
Parameter & Mean \pm SD \\
Age & $72.59 \pm 6.6$ \\
\hline BMI & $24.72 \pm 1.31$ \\
\hline Aneurysm sac diameter [mm] & $61.04 \pm 8.76$ \\
Parameter & $n(\%)$ \\
Diabetes mellitus & $4(18.1)$ \\
\hline Hypertension & $21(95.5)$ \\
\hline Hyperlipidaemia & $13(59)$ \\
\hline CAD & $13(59)$ \\
\hline Aneurysm type: & $20(91)$ \\
\hline Infrarenal & $2(9)$ \\
\hline Saccular &
\end{tabular}

$\mathrm{BMI}$ - body mass index, CAD - coronary artery disease. 

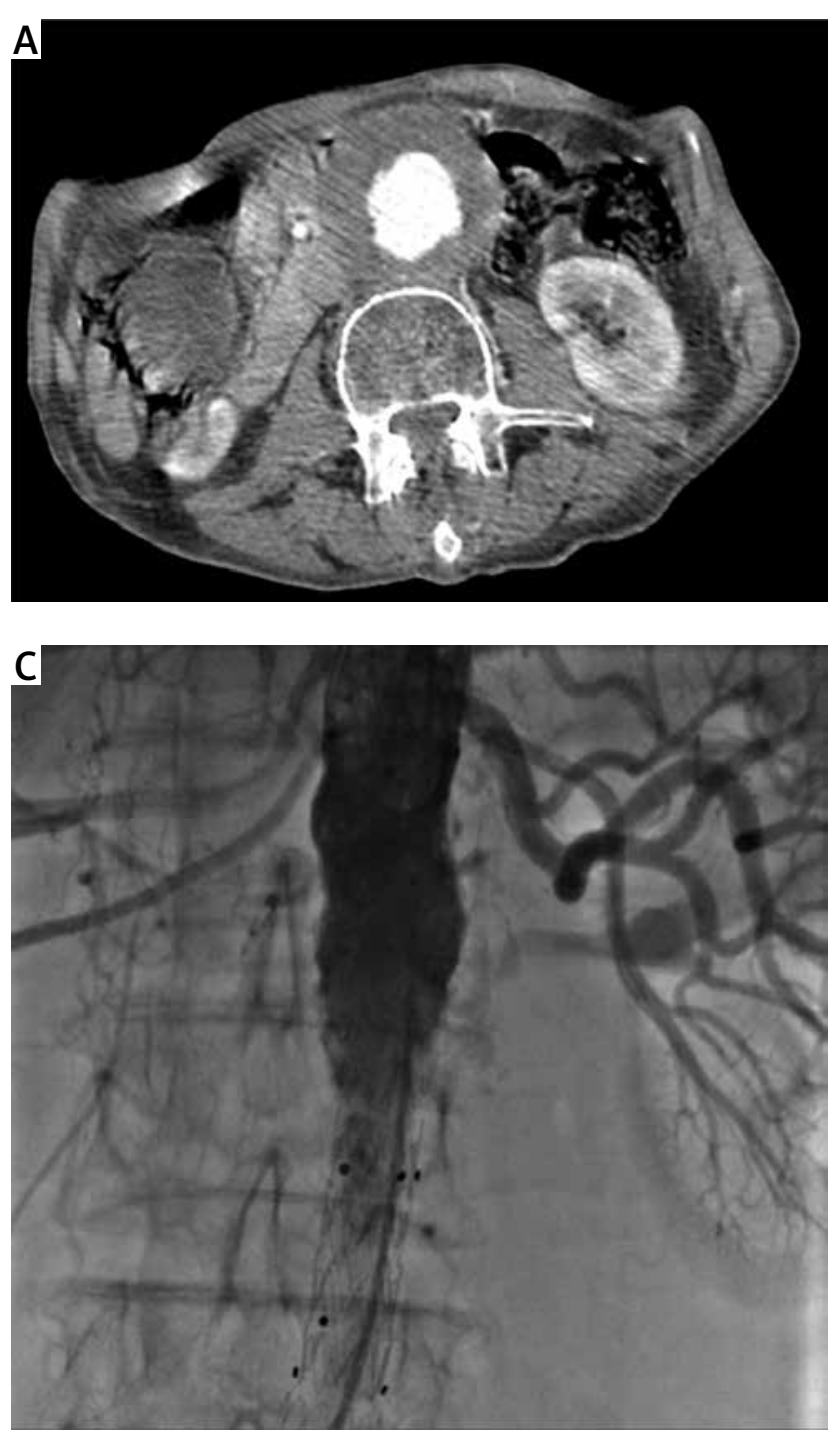

Figure 1. A, B - CT images of infrarenal AAA. C, D - Angiographic images following aorto-uni-iliac repair

Table II. Intraoperative characteristics of patients

\begin{tabular}{lc} 
Parameter & Mean \pm SD \\
Operative time [min] & $107.4 \pm 17.36$ \\
Parameter & $n(\%)$ \\
\hline \begin{tabular}{l} 
Endograft configuration: \\
\hline Bifurcated
\end{tabular} \\
\hline Aorto-uni-iliac & $1(95.5)$ \\
\hline Iliac extension: & $5(22)$ \\
\hline Right & $2(9)$ \\
\hline Left
\end{tabular}

\section{Discussion}

Parallel to the technological advances, the EVAR procedure has been defined as an alternative to surgical repair in AAAs and has been used more widely over the years [3-5].
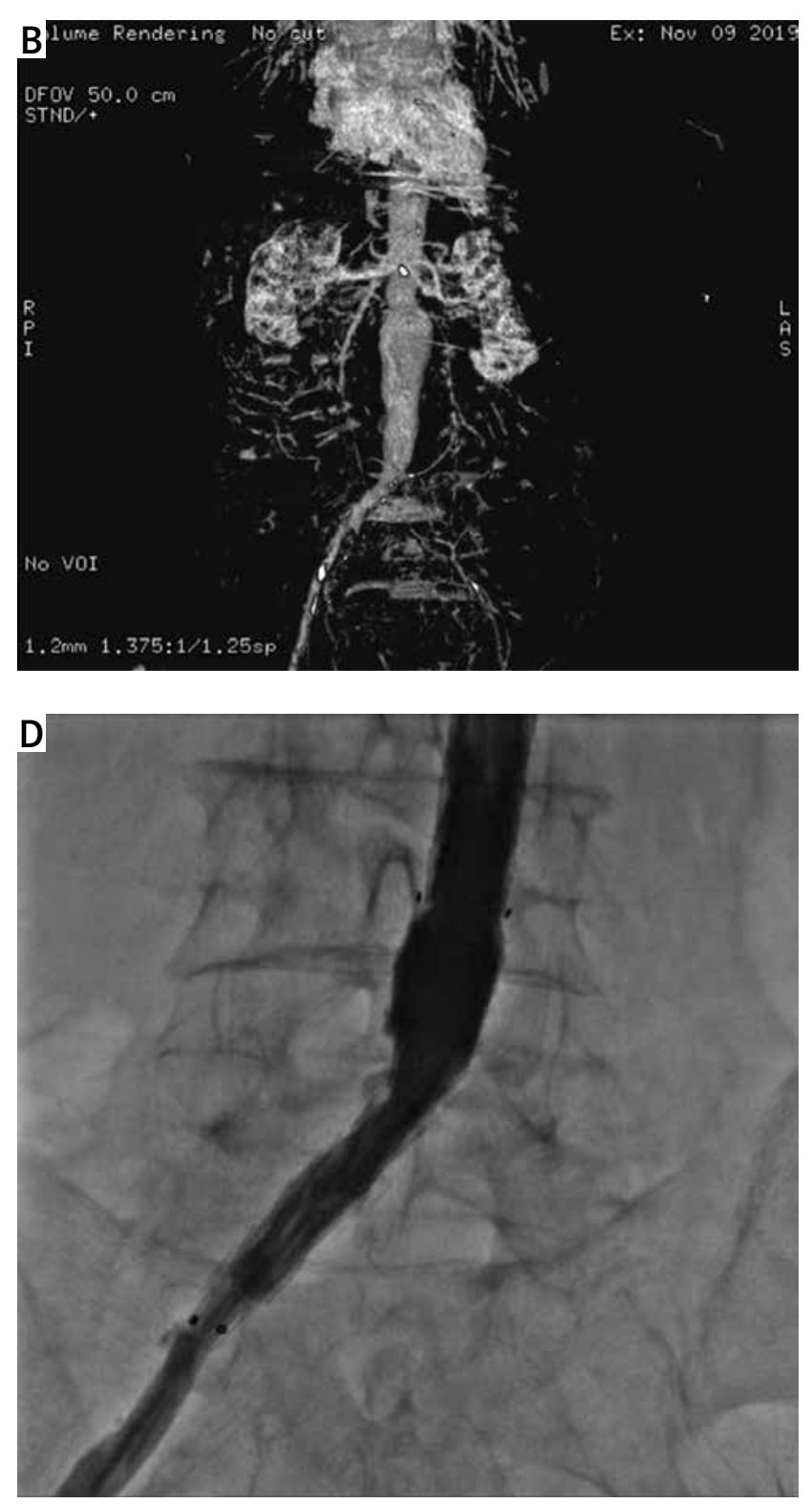

Although it is disadvantageous in terms of treatment costs, it is accepted as the first choice for a AAA repair due to its ease of application. In our clinic, we prefer to apply EVAR procedure as the first choice in all eligible AAA cases because of its known advantages.

As with all surgical procedures, there are multiple anaesthesia options available for EVAR procedures. However, there are many studies in the literature emphasizing the superiority of one anaesthesia technique over another. In a study by Cheng et al. it was emphasized that LA is advantageous in terms of operation time and hospital stay, and LA and RA are safe and effective options for elective EVAR cases [6]. In another study investigating the effect of anaesthesia type on perioperative outcomes, LA was associated with fewer pulmonary complications and it was stated that surgeons should consider the broader use of LA in appropriate EVAR cases [7]. As yet, there are no randomized trials comparing GA, RA, and LA. The multicentric, 
international ENGAGE study compared GA (62\%), RA (27\%), and LA (11\%). They concluded that anaesthesia preference did not affect perioperative morbidity and mortality, but locoregional anaesthesia decreased procedure time, ICU admission, and hospital stay [8].

Although LA is not a common type of anaesthesia in ruptured AAAs, it has been stated that LA may have a positive effect on EVAR outcomes compared to GA. This situation can be explained by the exacerbation of the haemodynamic effects of GA, including loss in vascular tone and decrease in bleeding, especially in patients presenting with shock [9]. In the study of Mouton et al. comparing the type of anaesthesia used during EVAR procedures in ruptured AAA patients, in-hospital mortality was reported to be lower in the LA group compared to the GA group (18.5\% vs. $28 \%$ ) [10]. We preferred to use LA in all cases, taking into account our clinical experience and current literature.

Endoleaks can be observed both in the early and the late postprocedural period. The incidence is reported to be as high as $25 \%$ during aneurysm repair. Luckily, some disappear without intervention, while others require immediate intervention to eliminate the risk of aneurysm rupture [11]. Endoleaks that occurred in our cases were intervened according to the current literature and guidelines.

It is known by all surgeons that keeping the incision size small in all surgical procedures with a minimalist approach provides advantages in terms of wound complications, patient comfort, and length of hospital stay. In this context, keeping the groin incision used during EVAR procedures small provides an advantage in terms of avoiding unnecessary injuries in this region rich in vascular and lymphatic network. In a Cochrane review comparing transverse and vertical groin incisions for femoral artery access, it was reported that fewer wound complications develop with the transverse incision, but this technique has some disadvantages, such as limitations regarding access to all vascular structures and increased injury to lymphatic vessels [12]. Based on this fact, we performed a limited vertical groin incision that was as small as possible to maintain a better vascular exposure in all our cases. Lymphocele developed in 2 patients within the first 15 days, which we treated with needle puncture, while haematoma was observed in 1 patient in the hospitalization period, which we revised under local anaesthesia.

Compared with open surgical repair, overall perioperative mortality for intact AAA repair with EVAR procedure is noticeably lower (1.1 to $1.5 \%$ vs. 3.9 to $4.4 \%$ ). This rate is very high for ruptured AAAs [13]. In the DREAM trial, in which EVAR and open surgical repair were compared, it was stated that EVAR was more advantageous in terms of hospital stay ( 6 days vs. 13 days) and 30-day mortality (1.2\% vs. 4.6\%). However, EVAR loses this early advantage in terms of mortality in long-term follow-up $[14,15]$. The length of hospital stay we achieved with the minimalist approach is very low, supporting the method we advocate. The high mortality rate in our patients presenting with ruptured AAA is attributed to the addition of cardiovascular comorbidities.
Table III. Postoperative characteristics of patients

\begin{tabular}{lc} 
Parameter & Mean \pm SD \\
Hospital stay [days] & $3.45 \pm 1.05$ \\
\hline Follow-up time [months] & $16.05 \pm 11.48$ \\
Parameter & $n(\%)$ \\
Endoleak: & $3(13.6)$ \\
\hline Type 1a & $1(4.5)$ \\
\hline Type 1b & $2(9)$ \\
\hline Type 2 & None \\
\hline Type 3 & None \\
\hline Type 4 & None \\
\hline Type 5 (Endotension) & $2(9)$ \\
\hline Lymphocele & $1(4.5)$ \\
\hline Haematoma & $2(9)$ \\
\hline In-hospital mortality & \\
\hline
\end{tabular}

In conclusion, because the EVAR procedure is an intervention that increases hospital costs compared to an open surgical repair, we think that choosing the type of anaesthesia and incision size with a more minimalist approach can reduce the length of hospital stay and minimize the complications that may occur after the procedure.

The study is of retrospective nature and small sized. A prospectively randomized trial comparing LA, RA, and GA would have given more accurate and projectile results.

\section{Acknowledgments}

The cases were performed in previous institute of the authors, Medical Park Uşak Hospital.

\section{Disclosure}

The authors report no conflict of interest.

\section{References}

1. Kothandan H, Haw Chieh GL, Khan SA, Karthekeyan RB, Sharad SS. Anesthetic considerations for endovascular abdominal aortic aneurysm repair. Ann Card Anaesth 2016; 19: 132-141.

2. Parodi JC, Palmaz JC, Barone HD. Transfemoral intraluminal graft implantation for abdominal aortic aneurysms. Ann Vasc Surg 1991; 5: 491-499.

3. Günes T, Yılık L, Yetkin U, Yürekli i, Ozcem B, Yazman S. A comparison of open conventional and endovascular surgical therapies in abdominal aortic aneurysm repair. Turk Gogus Kalp Dama 2012; 20: 515-523.

4. Yavuz Ş, Özbudak E, Gümüştaş S, Kanko M, Çitçi E, Berki T. Endovascular stent-graft applications in abdominal aortic aneurysms: mid- and long-term results. Turk Gogus Kalp Dama 2013; 21: 333-340.

5. Tayfur K, Ürkmez M, Yalçın M, Bademci ŞM, Gödekmerdan E, Koç A. Mid-term results of endovascular repair in isolated abdominal aortic aneurysms. Turk Gogus Kalp Dama 2015; 23: 274-279.

6. Cheng M, Chen Q, Tran-McCaslin M, Chun L, Lew W, Patel K. Endovascular abdominal aortic aneurysm repair: does anesthesia type matter? Ann Vasc Surg 2019; 61: 284-290.

7. Van Orden K, Farber A, Schermerhorn ML, Goodney PP, Kalish JAA, Jones DW, Rybin D, Siracuse JJ, Vascular Quality Initiative. Local anesthesia for percutaneous endovascular abdominal aortic aneurysm repair is associated with fewer pulmonary complications. J Vasc Surg 2018; 68: 1023-1029.e2. 
8. Broos PPHL, Stokmans RA, Cuypers PWM, van Sambeek MRHM, Teijink JAW. Effects of anesthesia type on perioperative outcome after endovascular aneurysm repair. J Endovasc Ther 2015; 22: 770-777.

9. Hope K, Nickols G, Mouton R. Modern anesthetic management of ruptured abdominal aortic aneurysms. J Cardiothorac Vasc Anesth 2016; 30: 1676-1684.

10. Mouton R, Rogers CA, Harris RA, Hinchliffe RJ. Local anaesthesia for endovascular repair of ruptured abdominal aortic aneurysm. Br J Surg 2019; 106: 74-81.

11. Chaikof EL, Dalman RL, Eskandari MK, Jackson BM, Anthony Lee W, Ashraf Mansour M, Mastracci TM, Mell M, Hassan Murad M, Nguyen LL, Oderich GS, Patel MS, Schermerhorn ML, Starnes BW. The Society for Vascular Surgery practice guidelines on the care of patients with an abdominal aortic aneurysm. J Vasc Surg 2018; 67: 2-77.

12. Canteras M, Baptista-Silva JC, do Carmo Novaes F, Cacione DG. Transverse versus vertical groin incision for femoral artery approach. Cochrane Database Syst Rev 2020; 4: CD013153.
13. Budtz-Lilly J, Wanhainen A, Mani K. Outcomes of endovascular aortic repair in the modern era. J Cardiovasc Surg 2018; 59: 180-189.

14. Prinssen M, Verhoeven EL, Buth J, Cuypers PWM, van Sanbeek MRHM, Balm R, Buskens E, Grobbee DE, Blankensteijn JD; Dutch Randomized Endovascular Aneurysm Management (DREAM)Trial Group. A randomized trial comparing conventional and endovascular repair of abdominal aortic aneurysms. N Engl J Med 2004; 351: 1607-1618.

15. Patel R, Sweeting MJ, Powell JT, Greenhalgh RM; EVAR Trial Investigators. Endovascular versus open repair of abdominal aortic aneurysm in 15-years' follow-up of the UK endovascular aneurysm repair trial 1 (EVAR trial 1): a randomised controlled trial. Lancet 2016; 388: 2366-2374. 